\title{
Calidad de vida, apoyo social y deterioro en una muestra de personas con trastorno mental grave*
}

\section{Quality of life, social support and impairment in a sample of persons with severe mental illness}

\author{
DANIEL NAVARRO BAYÓN ${ }^{1}$ \\ SUSANA GARCÍA-HERAS DELGADO ${ }^{2}$ \\ OLGA CARRASCO RAMÍREZ ${ }^{3}$ \\ ALBERTO CASAS TORAL ${ }^{2}$
}

Fecha de Recepción: 28-05-2007

Fecha de Aceptación: 04-11-2009

\section{RESUMEN}

La mayoría de las intervenciones en rehabilitación psicosocial tienen como objetivo principal mejorar el funcionamiento de la vida de las personas con trastorno mental grave para una mejor adaptación social, estando la calidad de vida relacionada con los tratamientos recibidos. El articulo evalúa los índices de calidad de vida, apoyo social percibido y deterioro psicosocial de una muestra de 89 sujetos con trastorno mental en contextos rurales y alejados de Castilla-La Mancha, atendidos desde el Programa de Integración social y apoyo comunitario ( PRIS ) de la Fundación FISLEM, que ofrece unos cuidados y seguimiento continuado que incluye el apoyo a procesos de rehabilitación psicosocial y laboral, soporte social, apoyo a la integración en su contexto y atención en el domicilio como complemento a los diferentes recursos de atención sociosanitaria a personas con enfermedad mental: centros de rehabilitación psicosocial y laboral y unidades de salud mental. Se ha encontrado relación entre la calidad de vida y otras variables estudiadas como el apoyo social ofrecido por el programa, el deterioro psicosocial y la situación sociolaboral.

\footnotetext{
${ }^{1}$ Psicólogo. Jefe de Programas de FISLEM.

${ }^{2}$ Trabajador social. Coordinadores del Programa Residencial de FISLEM.

${ }^{3}$ Psicóloga. Coordinadora del Programa de centros de Rehabilitación psicosocial y Laboral de FISLEM.

* El presente trabajo fue galardonado en la XI Convocatoria de FEAFES con el premio Jaime Albert Solana.
} 


\title{
PALABRAS CLAVE
}

Calidad de vida, Apoyo social, Trastorno mental grave, Rehabilitación psicosocial.

\begin{abstract}
The principal aims of the interventions in psychosocial rehabilitation is to improve the living functioning in persons with several mental illness, being the quality of life connected with the received treatments. The article evaluates the index of quality life, social support received and psychosocial deterioration in a sample of 89 subjects with mental illness in rural and removed areas of Castilla-La Mancha, attended by the Social Integration Community Support Program (PRIS) of FISLEM Foundation. This program offers care and follow up including integration and domestic attention as a complement to the different resources of sociosanitary attention to persons with mental illness: psychosocial and vocational rehabilitation centres and adult mental health services. The results confirm the relation between quality of life and other studied variables like social support offered by the program, psychosocial deterioration and the social and family situation.
\end{abstract}

\section{KEY WORDS}

Quality of life, Social support, Severe mental illness, Psychosocial rehabilitation. 


\section{INTRODUCCION}

La evaluación de resultados en el trastorno mental grave (TMG) y crónico debe basarse en indicadores que vayan más allá de las medidas clínicas tradicionales como la remisión de los sintomas o la prevención de recaídas (Chicharro y Uriarte, 2003). En la rehabilitación psicosocial las variables que afectan a la recuperación de los usuarios van más allá del diagnóstico, la gravedad de los síntomas y la adherencia al fármaco. Así, variables como apoyo familiar y social, autonomía económica, soporte residencial o dificultades en las actividades de la vida diaria influyen en la evolución y se convierten en dianas de la intervención.

En los trastornos mentales graves la definición del cuadro lleva asociada la cronicidad (Rodríguez y González, 2002; Blanco y Pastor, 1997), lo que supone la presencia de limitaciones en el funcionamiento y discapacidades en áreas como las habilidades de relación e integración social. Por eso, en la medición de resultados de los cuadros crónicos se necesitan medidas que permitan evaluar la relación con elementos relacionados con la reconstrucción social y la capacitación personal (Bowling, 1994). Aspectos relacionados en último término con los objetivos vitales de las personas: autonomía, apoyo social y calidad de vida.

Se han hecho varias propuestas para evaluar resultados en personas con TMG y para los diferentes servicios de rehabilitación psicosocial. Muñoz, Vázquez, Muñiz, López-Luengo, Hernangómez y Díaz (1999) proponen el uso de medidas de funcionamiento, satisfacción y bienestar, en concreto el Schizom (Cuffel, Fischer, Owen y Smith, 1997), el REHAB (Rehabiltiation Evaluation, Hall y Baker, 1983) y el Cuestionario de satisfacción general (López, 1994). Por otra parte, Liberman y Kopelwiz (2004) proponen una definición operativa de la recuperación de la esquizofrenia que incluye: remisión de sintomas, funcionamiento ocupacional, vida independiente y relaciones sociales.

Las propuestas anteriores están en relación con el acuerdo generalizado de los que trabajan en rehabilitación psicosocial de que la evaluación de resultados en el TMG debería abordar al menos dimensiones como: calidad de vida, satisfacción con los servicios, funcionamiento cotidiano y soportes sociales (Muñoz et al, 1994).

\section{CALIDAD DE VIDA, APOYO SOCIAL Y DETERIORO}

La mayoría de las intervenciones en rehabilitación psicosocial buscan como objetivo principal mejorar el funcionamiento de la vida de los usuarios para una mejor adaptación social. Dado que las personas con TMG viven cada vez más en la comunidad, los clínicos y los cuidadores argumentan que la calidad de vida debe estar relacionada con los tratamientos recibidos. Los estudios han indicado que los programas combinados de tratamiento farmacológico y rehabilitación disminuyen las recaídas, mejoran el funcionamiento y facilitan la integración social y laboral (Kingdom y Turkington, 2005; Vallina y Lemos, 2003; Liberman y Kopelwitz, 2004; Fallom et al, 2004). Sin embargo pocos estudios se han preocupado de medir si estos resultados positivos incluyen también mejoras en la calidad de vida, y los que se han hecho se han centrado fundamentalmente en pacientes que son dados de alta tras periodos largos de hospitalización (Lehman, Ward y Linn, 1982; Lehman, Possidente y Hawker, 1986). A pesar de eso, la calidad de vida se ha convertido en un concepto fundamental en la evaluación de los efectos de los tra- 
tamientos que se ofrecen a los pacientes crónicos.

Existen dos modelos de calidad de vida. Uno, utiliza medidas basadas en la satisfacción del individuo con áreas vitales como relaciones, familia, actividad recreativa, salud etc. (Argermeyer y Katsching, 1997). Se trata de una percepción subjetiva de la propia persona sobre un sentimiento de bienestar global y satisfacción con la vida. El otro enfoque se basa en medidas amplias que incluyen el funcionamiento, ingresos económicos, empleo, status residencial, etc. Se trata de una consideración de los aspectos objetivables del funcionamiento y adaptación del sujeto en las distintas áreas vitales. En general, existe un acuerdo en que la calidad de vida debe reflejar el bienestar de la persona tanto en su consideración objetiva como subjetiva.

En lo que se refiere al apoyo social hay evidencias de que a mayor apoyo social (AS), mayor estabilidad sintomatológica y permanencia en la comunidad en personas con TMG (Fakhoury, Murray, Shepherd y Priebe, 2002). Estos resultados concuerdan con el hecho de que el AS se ha reconocido como factor de protección de los problemas mentales. De hecho, la relación entre las puntuaciones en AS percibido y salud mental positiva es significativa en la mayoría de los países europeos. España presenta una de las correlaciones más altas (Ministerio de Sanidad y Consumo, 2007). Por otro lado, las personas con TMG suelen tener redes sociales de escaso tamaño, y el apoyo social suele estar restringido al ámbito de la familia o al propio sistema de cuidados sociales y sanitarios. Por eso no solo es importante intervenir en esta área, sino también proveer apoyos especificos directamente a través de los programas, en especial para las personas que viven en entornos con redes sociales pequeñas y poco diversas.

El curso de los TMG, y en especial de las esquizofrenias incluye desde una recuperación completa hasta un curso deteriorante prolongado durante años. Así, tras un tratamiento integral en la comunidad durante una media de dos años, aproximadamente el 50\% estará mejorado a los 5-6 años de seguimiento. El otro 50\% es probable que experimente lo que se ha denominado "curso crónico", que consiste en discapacidades o deterioros en lo social, personal y laboral (Hegarty, Baldessarini, Tohen, Waternaux y Oepen, 1994). En estudios de seguimiento longitudinal después del primer diagnóstico de esquizofrenia, se ha comprobado la importancia de la dimensión del deterioro en la etiqueta diagnóstica. Así, cuando se incluye una medida de funcionamiento se producen modificaciones en el diagnóstico a los diez años de revisión del mismo. Si las puntuaciones en el funcionamiento tienen una tendencia a la baja con los años, se ratifica el diagnóstico de esquizofrenia. A la inversa, cuando con los años las puntuaciones en funcionamiento tienden a subir se llega a eliminar la diagnostico inicial o a cambiarlo por otro (Bromet, Naz, Fochtmann, Carlson y Tanenberg, 2005). En estudios prospectivos de todo el mundo que van de 10 años a 25 años y que incluyen a más de 2.500 sujetos con esquizofrenia se llega a la conclusión de la presencia del deterioro como una dimensión definitoria de la gravedad asociada al trastorno. De hecho, se ha considerado tradicionalmente que la dimensión de funcionamiento opera independientemente de los sintomas (Shepherd, 1997).

El deterioro es la dimensión que más se utiliza para evaluar si una persona está o no está recuperada del trastorno (Harrow, Grossman, Jobe y Herbener, 
2005). Si el paciente muestra un incremento en autonomia personal, redes sociales, ocupación y trabajo, se considera como recuperado de las consecuencias negativas del trastorno. Utilizando la dimensión recuperación operativizada con medidas de funcionamiento se han encentrado porcentajes entre el 19\% y el $27 \%$ de personas con esquizofrenia recuperados de la enfermedad en seguimientos a 15 años (Harrow et al, 2005); Bromet et al, 2005) y hasta el $40 \%$ recuperados parcialmente (Bromet et al, 2005).

En lo que se refiere a los programas de intervención, se considera como imprescindible incluir programas psicosociales de tratamiento que complementen al tratamiento farmacológico, tanto en las guías de evidencia y práctica clínica (Emeterio et al, 2002) como en consensos de expertos (AEN, 2002) y en manuales prácticos de intervención (CAM, 2002).

\section{OBJETIVOS}

El objetivo es evaluar los índices de calidad de vida, apoyo social percibido y deterioro psicosocial en una muestra de sujetos con trastorno mental grave. El estudio forma parte de la evaluación de un programa de apoyo social comunitario de la Fundación Sociosanitaria para la Integración Social y Laboral del Enfermo Mental (FISLEM) de Castilla-La Mancha que incluye la descripción de la muestra, la satisfacción con la atención y el ajuste del programa a las características de los sujetos. También se examina la relación de las variables estudiadas con el sexo, diagnóstico, años de evolución y otros factores sociales y demográficos. Además se pretende conocer la incidencia o efectividad de la intervención en la evolución psicosocial, en concreto evaluando el nivel de deterioro antes y después de la intervención.

\section{METODO}

\section{Muestra}

La población seleccionada fueron los sujetos atendidos por el Programa de Integración Social y Apoyo Comunitario (PRIS) de FISLEM en el año 2006 y que hubieran recibido atención durante los años 2004 y 2005, durante un minimo de un año continuado. De todos los sujetos posibles se seleccionaron los que cumplian con los siguientes criterios de inclusión: tener un diagnóstico de enfermedad mental severa de larga evolución; residir en alguna de las áreas sanitarias de Castilla-La Mancha; tener entre 18 y 65 años; estar en tratamiento por una Unidad de Salud Mental (USM); haber tenido algún contacto con los dispositivos de salud mental de referencia en los dos años escogidos para el estudio; tener diseñado un plan individualizado de atención y seguimiento integrado dentro de un Centro de Rehabilitación Psicosocial y Laboral (CRPSL); coordinación con los profesionales del CRPSL y de la USM para el seguimiento del caso.

Se incluyeron 100 sujetos de los que se pudieron estudiar 89. Esta reducción viene explicada de la aplicación estricta de los criterios de inclusión y de incluir únicamente los usuarios atendidos por el programa en los años 2004 y 2005. Durante al año 2002 y 2003 se creó y definió el programa lo que conllevó una importante movilidad de usuarios como consecuencia del ajuste entre perfiles y actividades. De esta forma la muestra final presenta menos diferencias en variables clínicas, sociales y demográficas. Por tanto, el estudio se centra en una muestra concreta de usuarios atendidos durante un intervalo de tiempo, y no abarca a toda la totalidad de los que habian recibido atención desde el comienzo del programa. En la Tabla 1 se describen las características generales de la muestra. 
Tabla 1.

Caracteristicas generales de la muestra del estudio

\begin{tabular}{|c|c|}
\hline N Total & 89 \\
\hline Sexo h:m (\%) & $59(66): 30(34)$ \\
\hline Media de edad (d.típica) & $41(9.38)$ \\
\hline \multicolumn{2}{|l|}{ Rango de edad (\%) } \\
\hline $\begin{array}{l}18-29 \\
30-49 \\
>50\end{array}$ & $\begin{array}{l}10(11.4) \\
62(69.3) \\
17(19.3)\end{array}$ \\
\hline \multicolumn{2}{|l|}{ Diagnóstico } \\
\hline Esquizofrenia & $71.6 \%$ \\
\hline T.Bipolar & $11.4 \%$ \\
\hline T.Esquizoafectivo & $4.5 \%$ \\
\hline Depresión & $1.1 \%$ \\
\hline Ansiedad & $1.1 \%$ \\
\hline T.Personalidad & $8 \%$ \\
\hline \multicolumn{2}{|l|}{ Ingresos económicos } \\
\hline$<360$ euros & 50 (56.8) \\
\hline$>360$ euros & 35 (43.2) \\
\hline No tiene ingresos & $5(5.7)$ \\
\hline \multicolumn{2}{|l|}{ Minusvalia } \\
\hline$<65 \%$ & $10.2 \%$ \\
\hline$\geq 65 \%$ & $\mathbf{7 9 . 5} \%$ \\
\hline No & $9 \%$ \\
\hline Media edad del cuidador (D.T) & $65.5(13.1)$ \\
\hline Cuidadores con $>70$ años & $\mathbf{2 9}(38 \%)$ \\
\hline \multicolumn{2}{|l|}{ Situación laboral (\%) } \\
\hline Sin trabajo & 85 (95.4) \\
\hline Trabajando & $4(4.6)$ \\
\hline \multirow{2}{*}{ Media duración de la enfermedad (D.T.) } & $15.7(8.17)$ \\
\hline & \\
\hline$<2$ años & 3 (3.4) \\
\hline 2-10 años & 17(19.2) \\
\hline \multicolumn{2}{|l|}{$>10$ años } \\
\hline \multicolumn{2}{|l|}{ Domicilio } \\
\hline Rural & $62.5 \%$ \\
\hline Semirural & $\mathbf{2 1 . 6 \%}$ \\
\hline Urbano & $15.9 \%$ \\
\hline \multicolumn{2}{|l|}{ Distancia del domicilio al CRPSL } \\
\hline$<15 \mathrm{Km}$ & $21.5 \%(19)$ \\
\hline $15-30 \mathrm{Km}$ & $\mathbf{3 8 . 5} \%(\mathbf{3 4})$ \\
\hline$>30 \mathrm{Km}$ & $40 \%(35)$ \\
\hline \multirow{3}{*}{ Media meses de atención en el programa (D.T) } & $24.3(8.5)$ \\
\hline & Minimo 12 \\
\hline & Máximo 43 \\
\hline
\end{tabular}

Descripción del programa

Con el fin de encuadrar los resultados del estudio hay que exponer el obje- tivo y características de la intervención que reciben. De manera resumida, se trata de ofrecer a personas con TMG en contextos rurales y alejados, unos cui- 
dados y seguimiento continuado que incluye el apoyo a procesos de rehabilitación psicosocial y laboral, soporte social, apoyo a la integración en su contexto y atención en el domicilio. En la

\section{Medidas}

- Registro de datos del perfil de la muestra. Recoge datos descriptivos, clínicos, demográficos y sociales de

Tabla 2.

Caracteristicas del programa PRIS

- Asignación del caso a un profesional de referencia

- Trabajo sobre la población con ratio 1:15-20

- Integración en un equipo multidisplinar especializado en la rehabilitación psicosocial.

- Prestación de servicios en la comunidad

- Énfasis en el entrenamiento en habilidades y apoyos sociales

- Cobertura diuma, 5 dias a la semana

- Tratamiento coordinado con los servicios de salud mental de referencia

- Potenciación de la búsqueda de recursos sociales y laborales

- Plan individualizado de atención incluido dentro de un plan individual integral de tratamiento y rehabilitación

tabla 2 se resumen las características del programa.

Un profesional de referencia es asignado a cada caso para evaluar las necesidades, evitar la desconexión con los servicios de salud mental y mantener un asesoramiento continuado. La intervención la realiza un profesional denominado Técnico de Inserción Socio-laboral integrado en el equipo multidisciplinar de un CRPSL (psicólogo, trabajador social, terapeuta ocupacional y educador). Aunque el profesional es la referencia para el caso, el resto del equipo participa activamente en la toma de decisiones. El 75\% de su trabajo son intervenciones en el domicilio o en el medio, siendo el 25\% restante tiempo para reuniones de coordinación y planificación de actividades. Cada profesional del programa tiene asignados entre 15 y 20 casos. El trabajo que realizan se centra en ayuda práctica de la vida diaria, como parte de un plan individual de rehabilitación y seguimiento coordinado por el CRPSL y la USM. la muestra. Elaborado por FISLEM para el estudio.

- Apoyo social. No existe un consenso respecto al tipo de medida a utilizar, encontrándonos con un uso de cuestionarios y escalas específicamente diseñados para cada investigación (García-Cubillana, Luna, Laviana, Fernández, Perdiguero y López, 2006). La mayoría de las publicaciones hacen referencia al concepto cuantitativo del apoyo social, evaluando el número y frecuencia de las redes sociales, intentado delimitar las dimensiones más medibles del apoyo social. Sin embargo se dejan fuera dimensiones más subjetivas como las vivencias, funcionalidad o percepción que produce el contacto con la red social. Nos decidimos por buscar un instrumento que respondiera más a este último aspecto, menos investigado en la literatura actual. Escogimos el cuestionario de Apoyo Social DUKE-UNC. Está validada por 
Bellón, Delgado, Luna y Lardelli (1996). Evalúa la percepción subjetiva del apoyo percibido por el usuario en relación al programa de intervención en áreas como afecto, compañia, distracción, apoyo práctico, etc. Consta de 11 items y presenta una buena utilidad y facilidad para utilizarla en la práctica diaria de los equipos de intervención en contextos naturales.

- Calidad de vida. Se ha utilizado la Escala de Satisfacción con la Vida de Baker e Intagliata (1982). Evalúa la satisfacción del usuario en cuanto a su hogar, salud, vestuario, amigos, ocio, etc. Consta de 15 items y fue desarrollada con el objetivo de valorar el impacto de programas de apoyo comunitario en personas con TMG.

- Deterioro Psicosocial. El instrumento utilizado fue la Escala Breve de Evaluación de la Discapacidad (DAS, OMS, 1997). Recoge discapacidad asociada al trastorno mental en 4 áreas: autocuidados, ocupación, familia y red social. También recoge si el usuario precisa de supervisión en cada área de funcionamiento. La escala se pasó en dos momentos: antes de comenzar la intervención (pre) y en el momento de hacer la evaluación dos años después (post).

\section{Análisis de los datos}

Con el objetivo de mostrar las características básicas de la muestra en calidad de vida, apoyo social y deterioro, se realizaron análisis descriptivos a partir de las medidas de las puntuaciones. Para comprobar los cambios en el nivel de deterioro en los momentos pre y el post, se utilizó el test de Wilcoxon para comparar las puntuaciones del nivel de gravedad de la dis- capacidad, y la prueba de McNemar para comprobar si se habían producido cambios en la necesidad de supervisión en las áreas de funcionamiento. La correlación de Pearson se utilizó para ver el grado de relación entre la calidad de vida, el deterioro y el apoyo social. En este último análisis se utilizaron las puntuaciones obtenidas en la DAS en el momento de la evaluación (puntuaciones post), para comprobar si existía relación entre la reducción en el deterioro como consecuencia de la intervención y las otras dos variables. Para comprobar si existian diferencias significativas entre grupos de variables demográficas, clinicas y sociales (como sexo, diagnóstico, años de evolución y otras) se hicieron comparaciones de medidas con la t de Student o análisis de varianza según se tratara de variables con 2 ó más grupos de comparación. Se empleó el programa estadístico SPSS 12.0.

\section{RESULTADOS}

\section{Resultados en calidad de vida}

\section{Análisis descriptivos de la calidad de vida}

En la tabla 3 se pueden ver las puntuaciones medias en el cuestionario de calidad de vida. La media de la puntuación total en el cuestionario de calidad de vida es de 74,7 (d.t.=15).

Las puntuaciones más bajas están en las alternativas recreativas de su zona y respecto a su situación económica. Las más altas tienen que ver con aspectos instrumentales (comida y ropa).

\section{Análisis comparativos y de asociación en calidad de vida}

Se hicieron varios análisis para comprobar si existía una relación entre la 
Tabla 3.

Puntuaciones medias en el cuestionario de calidad de vida

\begin{tabular}{|c|c|c|}
\hline N= 82 usuarios con cuestionario completo ( $92 \%$ de la muestra) & $\begin{array}{l}\text { Media } \\
\text { (1 a 7) }\end{array}$ & D.T \\
\hline $\begin{array}{l}\text { 1. ¿Qué cara se acerca a cómo se siente con respecto a su vida } \\
\text { en general? }\end{array}$ & 4.8 & 1.6 \\
\hline $\begin{array}{l}\text { 2. ¿Qué cara se acerca a cómo se siente con respecto a su } \\
\text { hogar / piso / lugar de residencia? }\end{array}$ & 5.1 & 1.7 \\
\hline $\begin{array}{l}\text { 3. ¿Qué cara se acerca a cómo se siente con respecto a su } \\
\text { barrio como un sitio para vivir? }\end{array}$ & 4.9 & 1.8 \\
\hline $\begin{array}{l}\text { 4. ¿Qué cara se acerca a cómo se siente con respecto a la } \\
\text { comida que come? }\end{array}$ & 5.7 & 1.3 \\
\hline $\begin{array}{l}\text { 5. ¿Qué cara se acerca a cómo se siente con respecto a la } \\
\text { ropa que lleva? }\end{array}$ & 5.4 & 1.3 \\
\hline $\begin{array}{l}\text { 6. ¿Qué cara se acerca a cómo se siente con respecto a su } \\
\text { salud general? }\end{array}$ & 5.1 & 1.4 \\
\hline $\begin{array}{l}\text { 7. ¿Qué cara se acerca a cómo se siente con respecto a las } \\
\text { personas con que vive? }\end{array}$ & 5.7 & 1.3 \\
\hline $\begin{array}{l}\text { 8. ¿Qué cara se acerca a cómo se siente con respecto a sus } \\
\text { amigos? }\end{array}$ & 4.8 & 1.8 \\
\hline $\begin{array}{l}\text { 9. ¿Qué cara se acerca a cómo se siente con respecto a su } \\
\text { relación con su familia? }\end{array}$ & 5.5 & 1.4 \\
\hline $\begin{array}{l}\text { 10. ¿Qué cara se acerca a cómo se siente con respecto a su } \\
\text { relación con otras personas? (ni amigos, ni familia, ni } \\
\text { personas con las que vive) }\end{array}$ & 4.9 & 1.5 \\
\hline $\begin{array}{l}\text { 11. ¿Qué cara se acerca a cómo se siente con respecto a su } \\
\text { trabajo o actividad laboral? }\end{array}$ & - & - \\
\hline $\begin{array}{l}\text { 12. ¿Qué cara se acerca a cómo se siente con respecto a sus } \\
\text { actividades de ocio? }\end{array}$ & 5 & 1.5 \\
\hline $\begin{array}{l}\text { 13. ¿Qué cara se acerca a cómo se siente con respecto a los } \\
\text { servicios e instalaciones y las posibilidades de diversión en } \\
\text { su zona de residencia? }\end{array}$ & 4.3 & 1.8 \\
\hline $\begin{array}{l}\text { 14. ¿Qué cara se acerca a cómo se siente con respecto a los } \\
\text { servicios y equipamientos (hospitales, tiendas, } \\
\text { polideportivos, etc) en su zona }\end{array}$ & 4.7 & 1.7 \\
\hline $\begin{array}{l}\text { 15. ¿Qué cara se acerca a cómo se siente con respecto a su } \\
\text { situación económica? }\end{array}$ & 4.6 & 1.8 \\
\hline
\end{tabular}

calidad de vida y otras variables estudiadas como el apoyo social ofrecido por el programa, los años de evolución, el deterioro psicosocial y la situación sociolaboral. Para ello se realizaron medidas de comparación entre grupos y medidas de relación entre pares de variables.
Se ha encontrado una relación estadisticamente significativa entre la puntuación en calidad de vida y el apoyo social ofrecido por los profesionales, que indica que a mayor apoyo social percibido por el usuario, mejor calidad de vida (correlación de Pearson=0.3; $\mathrm{p}<0.01$ ). 
También se midió el grado de relación entre la calidad de vida y el número de ingresos psiquiátricos previos, no encontrándose ninguna relación entre dichas variables.

No se encontraron diferencias entre los años de evolución de la enfermedad y la calidad de vida total, lo que indica que esta no es una variable que tenga que ver con tener mayor o menor calidad de vida $(F=0.98 ; p=0.4)$. Sin embargo las personas de más años de evolución (>12 años) tienen menor puntuación en calidad de vida (media=73) que los que tienen menor duración de la enfermedad ( $<2$ años; media=92). Sin embargo las diferencias no son estadísticamente significativas y la distribución de la muestra entre los grupos de años de evolución tampoco es homogénea, por lo que no es algo concluyente.

No se encontraron diferencias en sexo $(\mathrm{H}=78$ vs $\mathrm{M}=73 ; \mathrm{t}=1.6 ; \mathrm{p}=0.11)$, ni en diagnóstico $(\mathrm{F}=1.2 ; \mathrm{p}=0.3)$. Sin embargo en la puntuaciones medias de calidad de vida en relación al diagnóstico se observan diferencias sobre todo entre dos grupos, los que tienen esquizofrenia (media=76) y los que tienen trastornos de la personalidad (media $=60$ ), a favor de los primeros.

No se encontraron diferencias en nivel de ingresos económicos ( $\mathrm{F}=0.3 ; \mathrm{p}=0.8)$, ni en situación laboral ( $\mathrm{F}=0.68 ; \mathrm{p}=0.6)$. Aunque las diferencias no son significativas, en el ultimo caso hay que mencionar que hay grupos que difieren de otros. Así, los que están trabajando tienen mejor calidad de vida que los que están parados, son pensionistas 0 están incapacitados (trabajando $=86$; pensionista $=75$; para$\mathrm{do}=72$; incapacitado $=75$ ). Debido a que en la muestra se encuentran muy pocos trabajando (tan sólo 4 sujetos) no se han podido encontrar diferencias significativas, aunque la diferencia entre los que están trabajando y los parados es de 14 puntos.

\section{Resultados en apoyo social}

\section{Análisis descriptivos del apoyo social}

En la tabla 4 se muestran las puntuaciones medias en el DUKE-UNC. La única puntuación por debajo de 4 es el apoyo percibido en relación a la posibilidad de hablar con alguien de los problemas económicos, aspecto que se relaciona con cierta insatisfacción en las puntuaciones anteriores en calidad de vida. El resto está por encima de 4 lo que refleja una percepción alta del apoyo en áreas como recibir elogios, compañia, distracción, o hablar sobre temas que preocupan.

\section{Análisis comparativos en apoyo social percibido.}

Para ver si existen diferencias en relación a las personas que viven solas 0 acompañadas se hizo el correspondiente análisis de varianza, no encontrándose diferencias significativas en la puntuación de la escala de apoyo social comparando las distintas categorias de convivencia (con padres, con madre, con padre, con hermanos, solo, cónyuge, en viviendas supervisadas). Aunque no son estadísticamente significativas se aprecia que los que viven solos perciben menor apoyo social por parte del programa (media $=45$, d.t. $=9.37$ ).

No se encontraron diferencias estadísticamente significativas entre las personas que viven en contexto rural, semirural o urbano. Hay que señalar que aunque las diferencias no son significativas si se encuentran entre los que viven en entornos rurales y urbanos, percibiendo mayor apoyo los primeros (rural $=49$, urbano $=46)$. 
Tabla 4.

Puntuaciones medias en el cuestionario de percepción de apoyo social

\begin{tabular}{|c|c|c|}
\hline $\mathrm{N}=81$ cuestionario analizados (91\% de la muestra) & $\begin{array}{l}\text { Media } \\
(1 \mathrm{a} \text { 5 })^{*} \\
\end{array}$ & D.T \\
\hline 1.- Recibo visitas y compañia & 4.4 & 0.9 \\
\hline 2.- Recibo ayuda en asuntos relacionados con mi casa & 4 & 1.12 \\
\hline 3.- Recibo elogios y reconocimiento cuando hago bien las cosas & 4.5 & 0.82 \\
\hline 4.- Cuento con personas que se preocupan de lo que me sucede & 4.5 & 0.92 \\
\hline 5.- Recibo afecto & 4.4 & 0.87 \\
\hline $\begin{array}{l}\text { 6. - Tengo la posibilidad de hablar con alguien de mis problemas en el trabajo } 0 \\
\text { en la casa }\end{array}$ & 4.2 & 1.01 \\
\hline $\begin{array}{l}\text { 7.- Tengo la posibilidad de hablar con alguien de mis problemas personales y } \\
\text { familiares }\end{array}$ & 4.3 & 1 \\
\hline 8.- Tengo la posibilidad de hablar con alguien de mis problemas económicos & 3.9 & 1.1 \\
\hline 9. Recibo ánimo y apoyo para distraerme y salir con otras personas & 4.3 & 1 \\
\hline $\begin{array}{l}\text { 10.- Recibo consejos útiles cuando me ocurre algún acontecimiento importante } \\
\text { en mi vida }\end{array}$ & 4.5 & 0.7 \\
\hline 11.- Recibo ayuda cuando estoy enfermo & 4.6 & 0.67 \\
\hline PUNTUAC & 48 & 5.82 \\
\hline
\end{tabular}

*Rango de puntuaciones

1: mucho menos de lo que deseo

2: menos de lo que deseo

3: ni mucho ni poco

4: casi como deseo

5: tanto como deseo

Se encontraron diferencias significativas entre las personas que viven a menor o mayor distancia del CRPSL, siendo los que viven más alejados (>30 Km) los que perciben un mayor apoyo del programa (media $=48 ;$ d.t. $=6.4$ ) y los que viven más cerca $(<15 \mathrm{Km})$ los que menor apoyo perciben del programa (media $=45$; d.t. $=6.15$ ).

No se encontraron diferencias entre los grupos de más o menos años de evolu- ción, ni en relación al tiempo que llevan en recibiendo la atención del programa.

\section{Resultados en deterioro}

1. Análisis Pre-post

Cambios en áreas de funcionamiento psicosocial al inicio de la intervención y en el momento de la evaluación 
Los resultados con el test de Wilcoxon de la tabla 5 indican que el cambio producido por la intervención es estadística-
2. Análisis de relación entre las áreas de deterioro psicosocial, el apoyo social y la calidad de vida

Tabla 5.

Comparaciones pre-post del nivel de deterioro en el funcionamiento

\begin{tabular}{lcccc}
\hline & $\begin{array}{c}\text { L.Base (pre) } \\
\text { Media (d.t.) } \\
\text { (0 a 5) }\end{array}$ & $\begin{array}{c}\text { Momento de la evaluación } \\
\text { (post*) }\end{array}$ & N & P \\
\hline DAS. Cuidado personal & $2.5(1.5)$ & $1.5(1.2)$ & 89 & P<0.001 \\
DAS Ocupación & $3.5(1)$ & $2.4(1.3)$ & 89 & P<0.001 \\
DAS Familia y hogar & $3.1(1.1)$ & $2.1(1.2)$ & 89 & P<0.001 \\
DAS Funcionamiento & $3.7(1)$ & $2.6(1.3)$ & 89 & $P<0.001$ \\
social & & & & \\
\hline * Todos los sujetos evaluados en el momento de la investigación llevan una \\
media de intervención de 24 meses en el programa.
\end{tabular}

mente significativo en todas las áreas de funcionamiento, reduciéndose el grado de discapacidad en cada área.

Se produce una reducción de la discapacidad del $40 \%$ en los autocuidados personales (higiene, vestido, hábitos de salud); una reducción del deterioro del $31 \%$ en el área de ocupación del tiempo libre; una reducción de $32 \%$ en el área de familia y hogar; y una reducción del $30 \%$ en el funcionamiento en contextos sociales. Todas las puntuaciones han bajado de una discapacidad seria-severa a una discapacidad moderada-ligera.

\section{Cambios en necesidad de asistencia (supervisión) en las áreas de funcionamiento del DAS al inicio de la intervención y en el momento de la evaluación}

Para comprobar si se han producido cambios en la necesidad de asistencia en las áreas de funcionamiento se utilizó una comparación pre-post con la prueba de McNemar para comparar porcentajes de cara a determinar si las diferencias entre los dos momentos de la evaluación son significativas. Los resultados están en la tabla 6.
Para comprobar si existía una relación entre las medidas objeto del estudio se hicieron análisis de correlación entre la DAS (usando puntuación post, como se ha explicado en el apartado de análisis), el cuestionario de calidad de vida y el DUKE-UNC. Los resultados están en la tabla 7.

Como se ve en la tabla anterior no existe una relación significativa entre el deterioro y el apoyo social percibido. Si que existe una relación inversa estadísticamente significativa entre deterioro y calidad de vida. Mientras menor sea el deterioro en las áreas de ocupación, familia y funcionamiento social, mayor calidad de vida se tiene. El mayor nivel de relación con la calidad de vida se encuentra en el área de deterioro en familia-hogar. Estos resultados concuerdan con otros estudios que demuestran una fuerte relación entre la calidad de vida y el nivel de funcionamiento (Malla, Williams, Kopala, Smith, Talling y Balshaw, 2006); (Mercier y King, 1994). Aunque la relación entre el deterioro y el apoyo social no es significativa, es inversa, lo que indica que a menor discapacidad mayor apoyo se percibe. Sin embargo esta relación no es estadisticamente significativa. 
Tabla 6.

Comparaciones pre-post de la necesidad de supervisión en las áreas de funcionamiento

\begin{tabular}{|c|c|c|c|c|}
\hline & L.Base (pre) & $\begin{array}{c}\text { Momento de la evaluación } \\
\text { (post*) }\end{array}$ & $\mathbf{N}$ & $\mathbf{P}$ \\
\hline DAS. Cuidado personal & $\begin{array}{l}71 \%^{\mathrm{a}} \\
29 \%^{\mathrm{b}}\end{array}$ & $\begin{array}{l}70 \%{ }^{a} \\
30 \%\end{array}$ & 89 & N.S \\
\hline DAS Ocupación & $\begin{array}{l}74 \%^{a} \\
26 \%^{\text {b }}\end{array}$ & $\begin{array}{l}\mathbf{7 5} \%^{\mathrm{a}} \\
\mathbf{2 5} \%^{\mathrm{b}}\end{array}$ & 89 & N.S \\
\hline DAS Familia y hogar & $\begin{array}{l}84 \%^{a} \\
16 \%^{b}\end{array}$ & $\begin{array}{l}84 \%^{a} \\
16 \%\end{array}$ & 89 & N.S \\
\hline $\begin{array}{l}\text { DAS Funcionamiento } \\
\text { social }\end{array}$ & $\begin{array}{l}\mathbf{7 7} \%^{\mathrm{a}} \\
\mathbf{2 3} \%^{\mathrm{b}}\end{array}$ & $\begin{array}{l}72 \%^{\mathrm{a}} \\
\mathbf{2 8} \%^{\mathrm{b}}\end{array}$ & 89 & N.S \\
\hline
\end{tabular}

* Todos los sujetos evaluados en el momento de la investigación llevan una media de intervención de 24 meses en el programa.

a. Porcentaje de sujetos con funcionamiento sin asistencia; b. Porcentaje de sujetos con funcionamiento con asistencia

N.S. Diferencias no significativas.

Tabla 7. C

Correlaciones de Pearson entre deterioro, apoyo social y calidad de vida.

\begin{tabular}{lcccc}
\hline & DUKE-UNC $^{\natural}$ & P & C.VIDA $^{2}$ & P \\
\hline DAS. Cuidado personal & -0.18 & N.S & 0 & N.S. \\
DAS Ocupación & -0.14 & N.S & -0.22 & P<0.05 \\
DAS Familia y hogar & -0.1 & N.S & -0.3 & P<0.05 \\
DAS Funcionamiento social & 0 & N.S & -0.25 & P<0.05 \\
\hline
\end{tabular}

a. Coeficiente de correlación de Pearson

\section{Análisis comparativos en deterioro}

No se encontraron diferencias significativas en deterioro en el funcionamiento en cuanto a ser hombre o mujer, ni en relación a la duración de la enfermedad, ni en el tipo de núcleo de convivencia.

Si se encontraron diferencias significativas en los diagnósticos. Las personas con trastorno de personalidad tienen más discapacidad en las áreas de funcionamiento en el hogar y familia $(\mathrm{F}=4.6$; $\mathrm{p}<0.001)$, y en el funcionamiento social $(\mathrm{F}=2.5 ; \mathrm{p}<0.05)$ en comparación con cualquiera de los otros diagnósticos (puntuaciones medias en familia y hogar: esquizofrenia 2; bipolar 1.4; depresión 2; ansiedad 1; trastorno de personalidad 3.7. Puntuaciones medias en funcionamiento social: esquizofrenia 2.5; bipolar 2; depresión 3; ansiedad 3; trastorno de personalidad 3.7).

Para comprobar si hay relación entre la carga percibida por el cuidador y el deterioro psicosocial, se hicieron comparaciones mediante tablas de contingencia, encontrándose que los sujetos que tienen menos deterioro en el área de ocupación pertenecen al grupo de cuidadores que menor carga perciben en relación a sus ocupaciones y tiempo libre $\left(\mathrm{Chi}^{2}=16.7\right.$; g.l=15; $\left.\mathrm{p}<0.01\right)$. Lo que indi- 
ca que mientras el usuario tenga más actividades en que ocupar su tiempo, también es así para el cuidador principal.

\section{CONCLUSIONES}

Los datos demuestran que debe hacerse énfasis en aspectos como el trabajo "en vivo" en el entorno del usuario, potenciando su adaptación al medio social, haciendo hincapié en la creación de una adecuada alianza terapéutica y dando especial importancia a las intervenciones directamente sobre la red social y familiar del paciente.

Consideramos que la percepción de menos apoyo en el área económica puede tener que ver con las expectativas de cambio en la situación económica por parte de todos los implicados en el proceso de rehabilitación. Seria interesante poder realizar un estudio en profundidad de este aspecto

A pesar de la importancia de las cuestiones más básicas como la compañia, los resultados en el deterioro nos indican la necesidad de que existan otras intervenciones complementarias que aseguren una mejora en el funcionamiento psicosocial, ya que la relación terapéutica y el empleo de refuerzo por sí sólo no parece implicar un cambio en los niveles de desempeño.

La mayoría de los sujetos de la muestra, se encuentran en un entorno rural y alejado de los CRPSL (67\%). Los resultados en cuanto al apoyo social, indican que las personas más alejadas $(>30 \mathrm{Km})$ de los CRPSL perciben más apoyo del programa que los que viven más cerca $(<15 \mathrm{Km})$. Serían necesarios estudios específicos, que puedan ofrecernos datos sobre la relación entre la distancia, asistencia a CRPSL y apoyo social. Podríamos pensar, que aquellos que viven más cerca, también pueden asistir con más frecuencia a otras actividades ofrecidas por el CRPSL y por lo tanto cuentan con mayor número de apoyos y más variados, no centrados exclusivamente en la figura del técnico de referencia, que en los casos de personas más alejadas podría funcionar como único apoyo.

Los resultados indican niveles más bajos de calidad de vida en relación a los servicios y equipamientos que le ofrece su zona. Esto requiere un esfuerzo importante por parte de los técnicos para enfocar sus intervenciones hacia la creación y fomento de alternativas especificas que faciliten la integración comunitaria y el contacto social de los usuarios. Al margen de que los contextos rurales pueden caracterizarse por la escasez de recursos sociales y de ocio, las bajas puntuaciones en cuanto a las posibilidades de diversión en su zona no implican peores puntuaciones en calidad de vida respecto a las actividades de ocio que los pacientes desarrollan. Se debería profundizar en la representación que los pacientes tienen de su ocio y el tipo de actividades concretas que realizan, manteniendo una linea de intervención que potencie las oportunidades culturales, lúdicas o de contacto social desde el trabajo coordinado con los recursos disponibles en la zona, e incrementando la consideración de la red que tienen los usuarios.

Los resultados también indican una menor percepción de apoyo social de los usuarios que viven solos frente a los que conviven con familiares. Hipotetizamos que los efectos de nuestras intervenciones pueden influir en la mejora de la relación de convivencia y en la reducción de niveles elevados de carga familiar, mejorando el ambiente familiar y las actitudes que los familiares muestran hacia el paciente. Por otro lado los familiares podrian actuar como facilitadores 
en el recordatorio de las pautas marcadas e intervenciones diseñadas con el paciente, mientras que los que viven solos exclusivamente contarían con el apoyo del programa para la puesta en práctica y mantenimiento de los aprendizajes adquiridos.

La mayor relación entre la calidad de vida y el deterioro en el área familiar plantea la necesidad de incorporar intervenciones enfocadas hacia la reducción del estrés y carga familiar, el aprendizaje de habilidades para la solución de problemas y de modos de afrontamiento eficaces que permitan el manejo de determinadas situaciones conflictivas que se dan en la convivencia diaria.
Otras cuestiones que podrian ser analizadas en futuros estudios, serían comprobar la relación de otras variables características de la intervención del programa con las medidas de calidad de vida, apoyo social y deterioro, como puede ser, la frecuencia de contactos de los técnicos con el usuario o determinadas características o cualidades de los profesionales en cuanto a la relación que establecen con los pacientes, que pueden ser criticas a la hora de potenciar un cambio (conocimiento de las necesidades del colectivo y de las oportunidades del contexto, experto en el uso o aplicación de técnicas específicas, capacidad para transmitir reforzamiento positivo, disponibilidad, etc. ).

\section{BIBLIOGRAFIA}

AEN. (2002). Rehabilitación Psicosocial del Trastorno Mental Severo. Situación actual y recomendaciones. Madrid: Cuadernos Técnicos no 6 . Asociación Española de Neuropsiquiatria.

Argermeyer, M., C. y Katsching, H. (1997). Theoretical models of quality of life for mental disorders. En Katsching, H., Freeman H., Sartorius N (Eds.): Quality of life in mental disorders. 3-15. New York: Willey.

Baker, F. e Intagliata, J. (1982). Quality of Life in the evaluation of community support systems. En G. Bobes, M. Portilla y M.Garcia (1995). Calidad de vida en las Esquizofrenias. J.R Prous Editores.

Baker, R. y Hall, J., N. (1983). Rehabilitation Evaluation of Hall and Baker (REHAB). Aberdeen:Vine Publishing Ltd.

Bellón, J., A., Delgado, A., Luna, J. y Lardelli, P.(1996). Validez y fiabilidad del cuestionario de apoyo social Duke-UNC. Aten Primaria, 18, 153163. Extraído de Servicio Andaluz de Salud: "Cuestionarios, test e índices para la valoración del paciente en salud mental" (2004), Consejería de Salud.
Blanco, A. y Pastor, A. (1997). Las personas con enfermedades mentales crónicas: delimitación problemática y necesidades. En Rodríguez, A. (Comp.): Rehabilitación Psicosocial de personas con trastornos mentales crónicos, 33-55. Madrid: Pirámide.

Bowling, A. (1994). La medida de la Salud. Revisión de las escalas de medida de la calidad de vida. Barcelona: Masson S.A.

Bromet, E., J., Naz, B., Fochtmann, L., J., Carlson, G. y Tanenberg, M. (2005). Long term diagnostic stability and outcome in recent frist-episode cohort studies of schizophrenia. Schizophrenia Bulletin, 31 (3), 639-649.

CAM. (2002). Rehabilitación Psicosocial y apoyo comunitario de personas con enfermedad mental crónica: programas básicos de intervención. Madrid. Cuadernos Técnicos de la Consejería de servicios Sociales no 17 y 14. Comunidad de Madrid.

Chicharro, F. y Uriarte, J. (2003). Gestión clínica y rehabilitación psicosocial. Los nuevos modelos de gestión y la asistencia a la enfermedad mental 
severa. En C. G. Aguilar (Ed.): Rehabilitación Psicosocial y tratamiento integral del trastorno mental severo (231-273). AEN: Estudios.

Cuffel, B., J., Fischer, E., P., Owen, R., R. y Smith, G, .R. (1997). An instrument for measurement of outcomes of care for schizophrenia. Evaluation and the health professions, 20.96-108.

Emeterio et al. (2004).Guía de práctica clínica para la atención al paciente con esquizofrenia Barcelona: aatrm.

Fakhoury, W., K., Murray, A., Shepherd, G. y Priebe, S. (2002). Research in supported housing. Social Psychiatry Epidemiology, 37, 301-315.

Fallon et al. (2004).Tratamientos basados en la evidencia en la Esquizofrenia: resultados de dos años de seguimiento en un ensayo clínico internacional de campo sobre el tratamiento óptimo. World Psychiatry, 2:2 Sept, 104-109.

García-Cubillana, P., Luna, J., Laviana, M., Fernández, L., Perdiguero, D. y López, M. (2006) Adaptación y fiabilidad del cuestionario de red social, versión española del "Questionario Sulla Rete Sociale”, Aplicado a personas con trastorno mental severo. Revista de la AEN, 97, 135-150.

Harrow, M., Grossman, L., Jobe, T. y Herbener E. (2005). Do patients with schizophrenia show periods of recovery?. A 15 year multi-follow-up study. Schizophrenia Bulletin, 31 (3), 723-734.

Hegarty, J., D., Baldessarini, R., J., Tohen, M., Waternaux, C. y Oepen, G. (1994). One hundred years of schizophrenia: a meta-analysis of the outcome literatura. American Journal of Psychiatry, 151, 1409-1416.

Kingdon, D. y Turkington, D. (2005). Evidence for effective treatments in schizophrenia. En: Kingdon y Turkington: Cognitive Therapy of Schizophrenia.(28-42).New York: Guilford Press.

Lehman, A., F., Possidente, S. y Hawker, F. (1986). The quality of life of chronic patients in a State Hospital and in Community Residences. Hos pital and Community Psychiatry, 37(9), 901-907.

Lehman, A., F., Ward, N., C. y Linn, L.. (1982). Chronic mental patients: the quality of life issue. American Journal of Psychiatry, 139(10), 12711276.
Liberman, R., P. y Kopelwiz, A. (2004). Un enfoque empírico de la recuperación de la esquizofrenia: definir la recuperación e identificar los factores que pueden facilitarla. Rehabilitación Psicosocial, 1, $12-29$

López, A. (1994). Valoración de los CRPS de la CAM. Universidad de Málaga: Tesis Doctoral.

Malla, A., Williams, R., Kopala, L., Smith, G., Talling, D. y Balshaw, R. (2006). Outcome in quality of life in a Canadian nacional sample of patients with schizophrenia and realted psychotic disorders. Acta Psychiatrica Scandinavica, 113(Suppl.430), 22-28.

Mercier, C. y King, S. (1994). A latent variable causal model of the quality of life and community tenure of psychotic patients. Acta Psychiatrica Scandinavica, 89, 72-77.

Ministerio de Sanidad y Consumo (2007). Estra tegia en salud mental del Sistema Nacional de Salud. Madrid.

Muñoz, M., Vázquez, C., Muñiz, E., LópezLuengo, B., Hernangómez, L. y Díaz, M. (1999). Evaluación de resultados en la esquizofrenia: un ejemplo de aflicción en al comunidad autónoma de Madrid. Intervención Psicosocial, 1, 73-87.

Organización Mundial de la Salud (1997) Escala de evaluación de la Discapacidad WHODAS.

Rodríguez, A. y Cases J., C. (2002). La Rehabilitación Psicosocial en el marco de la atención comunitaria integral a la población enferma mental crónica. 17-42. En: Cuadernos Técnicos de Servicios sociales №17: Rehabilitación Psicosocial y apoyo Comunitario de personas con enfermedad mental crónica: programas básicos de intervención. Consejería de Servicios Sociales: Madrid.

Shepherd, G. (1997). Avances recientes en la rehabilitación psiquiátrica. En J. A. Aldaz y C. Vázquez (Eds.): Esquizofrenia: fundamentos psicológicos y psiquiátricos de la rehabilitación. 1-22. Edit: Siglo XXI.

Vallina, O. y Lemos, S. (2003). Guía de tratamientos psicológicos eficaces para la esquizofrenia. En: M. Alvarez, J. R. Hermida, C. Rodríguez y I. A.Vázquez (Eds.): Guía de tratamientos psicológicos eficaces I. Adultos.35-79. Madrid: Pirámide. 